\title{
La filière du cocotier au Ghana et au Nigéria
}

\author{
Serge BENE ${ }^{1}$ \\ Philippe COURBET ${ }^{2}$ \\ Ambruse DZIWORMU ${ }^{2}$ \\ Jérémy SALINIER ${ }^{3}$ \\ Akwassi MENSAH-BONSU \\ ${ }^{1}$ Consultant, I\&D/Bourbon Madagascar, \\ Services aux agriculteurs \\ <sbene@moov.mg> \\ ${ }^{2}$ Université du Ghana, Legon, Accra, Ghana \\ ${ }^{3}$ Institut des régions chaudes (IRC), \\ 1101, avenue Agropolis CS 24501, \\ 34093 Montpellier cedex 05, France
}

\begin{abstract}
In last ten years (1998-2007), the area planted to coconut in the world (average $12 \mathrm{Mha}$ ) has shown a decreasing trend (average 1\%/year). World coconut oil production has remained at more or less the same level in the last ten years (average $3 \mathrm{MT} /$ year). The study of the coconut commodity chain in Chana show that this sector is confronted with many constraints: the inadequacy price of coconut oil and the competition with other oleaginous plants; an aging coconut plantation; the impacts of the coconut yellow lethal disease, an important demand in peeled nuts towards Nigeria market and the weak dynamism of its institutional organization. Four commodity chains are moving together: the commodity chains of the processing of traditional and semi-industrial coconut oil produce little added value. The commodity of fresh nuts produces a higher added value with limited urban market. Finally, the recent export of peeled nuts commodity chain towards Nigeria offers a large market and brings an important added value to the smallholders. Despite a replanting project in hybrid coconuts, the rehabilitation/replanting of coconut plantation remains one of the challenges to deal with, in order to boost the coconut commodity chain in Ghana. The observation is that smallholders have not taken up the challenge after the public interventions have seized, in order to rehabilitate or replant their coconut trees. The strategy developed does not sufficiently lay on the market signal which today has evolved. The improved material available and the tested technologies should allow the boosting of the production sector and the reduction of the vulnerable conditions of household affected or threaten by the disease. The same way, the divergent strategies of stakeholders of the coconut commodity chain in Ghana slow down the dynamic of reorientation of this sector towards the export of peeled nuts. Producers in particular, should take advantage of this lucrative commodity chain. It is about all the stakeholders having interest in this commodity chain by preserving the coconut oil sector, which shows an economic, but also considerable social dimension. The boost of this production passes necessarily through the support function of the production and through professional organizations of producers.
\end{abstract}

Keywords: commodity chain analysis, coconut oil, fresh nuts, professional organization, Ghana, Nigeria
Le cocotier est une culture largement implantée dans les systèmes de production familiaux de la zone côtière du Ghana et fut longtemps le moteur du développement économique de cette région. Cette spéculation a offert, depuis au moins 80 ans, la possibilité de mettre en valeur une bande littorale de quelques kilomètres de largeur, ayant une hygrométrie très élevée, des sols sableux, et donc peu propice à d'autres cultures. La production des cocotiers revêt pour les ménages d'agriculteurs de cette frange littorale un intérêt triple : consommation familiale, trésorerie « sur tronc » (on vend des noix quand on a besoin d'argent) ou garantie pour les crédits (la production est gagée en échange d'un crédit octroyé pour résoudre des besoins ponctuels) et source de revenu monétaire [1]. Les cocoteraies font partie intégrante du patrimoine familial et se transmettent de génération en génération. Les cocoteraies au Ghana représentent environ 36000 ha, dont 12000 ha dans la principale zone de produc- tion de Jomoro (Western Region). Bien que le secteur cocotier mobilise beaucoup de main$d^{\prime} œ u v r e$ et d'activités induites au niveau local (collecte, cracking, transport, transformation, commerce, etc.), sa contribution au PIB s'avère faible. La filière noix de coco se caractérise également par la cohabitation de quatre sousfilières aujourd'hui en situation de concurrence : huile de coco, noix débourrées, coprah et noix fraîche.

La maladie du jaunissement mortel du cocotier s'est déclarée au Ghana en 1932, au Cap SaintPaul dans la région Volta (est du pays). Cette maladie s'est progressivement étendue: en 1964 dans la zone du Cape Three Points, puis à partir de 1986 dans la Central Region et la Western Region (ouest du pays). Des milliers d'hectares de cocoteraies ont été décimés, et le capital des producteurs a été anéanti. Entre 1999 et 2003, un projet financé par l'AFD propose aux producteurs l'adoption d'un hybride supposé résistant à la maladie. L'intérêt des producteurs à replanter a été assez faible, d'une part, parce que cette activité était à l'époque faiblement rémunératrice (comparativement à l'hévéa et au palmier à huile), d'autre part, en raison des risques phytosanitaires importants.

Jusqu'à une période récente, le secteur du cocotier n'était plus reconnu par les autorités comme un secteur économique important, comparé aux autres cultures de rente et pérennes: cacao, palmier à huile, hévéa, agrumes, etc. Le soutien du Ministry of Food and Agriculture (MOFA) et de son partenaire (Agence française de développement) au développement du secteur cocotier s'est achevé en 2006, sans nouvelles perspectives. Au final, les différents acteurs de la filière $s^{\prime}$ interrogent sur le devenir de leurs activités.

Le facteur prix au producteur est primordial pour relancer la replantation ou la réhabilitation des cocoteraies et l'entretien des plantations. Ce paramètre prix a très sensiblement évolué au cours de l'année 2006 avec l'arrivée 
en masse de marchands nigérians au Ghana pour acheter des noix débourrées (les acheteurs nigérians ont commencé à travailler au Ghana en 2002). Cette brutale augmentation a contribué à faire doubler le prix des noix débourrées en 2006 [1, 2]. Le marché de la noix de coco devrait donc peu à peu se redéployer vers cette sous-filière à forte demande. Les prix des noix débourrées sont aujourd'hui quatre à cinq fois supérieurs à ceux du premier semestre 2006. Sur le terrain, les quatre sousfilières cohabitent toujours, mais l'attractivité du marché nigérian compromet l'avenir des secteurs huile de coco et coprah. Aujourd'hui, le secteur noix de coco au Ghana reste confronté à un double enjeu : d'une part, développer son niveau de production et, d'autre part, améliorer l'organisation de la filière et du marché pour optimiser le niveau de valeur ajoutée produite localement $[1,7]$.

Dans cette communication, il s'agit de présenter les caractéristiques des sous-filières présentes au Ghana et d'essayer de répondre à ces questions: quelles sont la ou les filière(s) favorable(s) à la relance de ce secteur économique délaissé par l'État ghanéen? Et par quels moyens?

\section{Présentation du secteur cocotier au Ghana}

\section{Passage d'une culture d'exportation vers une culture de production d'huile par les petits producteurs}

Au début $d u x x^{\mathrm{e}}$ siècle, la culture du cocotier était principalement destinée à l'exportation du coprah vers les usines britanniques qui produisaient une huile utilisée dans la savonnerie. À partir de 1920, pour augmenter les exportations, le département de l'agriculture encouragea le développement des plantations villageoises, qui se répandirent peu à peu sur toutes les côtes du Ghana. Pour ce faire, les propriétaires fonciers ont cédé des parcelles en métayage à des agriculteurs souvent migrants, à travers le système de l'Abusa : c'est-à-dire qu'ils donnaient un terrain vierge à quelqu'un qui devait y installer une cocoteraie. Quand les plantations commençaient à produire, un tiers de la récolte revenait au propriétaire, le métayer gardait les deux tiers restants. Ce système, certes en "perte de vitesse ", est encore utilisé aujourd'hui pour la plantation des cultures pérennes. Traditionnellement, les villageois utilisaient l'huile de palme rouge comme base protéique de leur alimentation. Mais, avec le développement du cocotier, les habitants ont appris à en tirer de l'huile de friture de façon manuelle. Cette filière est devenue concurrente de la filière d'exportation de coprah. Les Britan- niques ont essayé de maintenir la production de coprah par un soutien des prix. Cette politique a été compromise par l'arrivée de la maladie du jaunissement mortel du cocotier, en 1932, dans la région Volta. La production d'huile de coprah a été fortement freinée. Toutefois, dès l'indépendance, le nouveau gouvernement ghanéen a essayé de soutenir cette sous-filière en créant à Esiama, en 1959, une usine d'État ${ }^{1}$ de transformation du coprah en huile (Industrial Development Company). L'arrêt progressif des importations britanniques, dans les années 1960, a contraint l'usine à réduire son activité et les planteurs à davantage destiner leur production aux marchés locaux (huile alimentaire de coco) ou à leur propre consommation. Cependant, la cohabitation des secteurs huile de coco (traitement par voie humide) et huile de coprah (traitement par voie sèche) a favorisé l'extension de la cocoteraie grâce au fort développement des plantations en Western Region (années 1960), où le climat humide est très propice aux cocotiers. Cette région devient le principal centre de production ghanéen. Cette dynamique va être perturbée à partir de 1964 par l'arrivée de la maladie du jaunissement mortel. Aujourd'hui, I'aire indemne de maladie se limite au district de Jomoro et à la partie ouest de celui de Nzema East. Les régions affectées par la maladie n'ont plus assez de noix pour la transformation en huile, ce qui pénalise l'ensemble de la filière de transformation (figure 1).

Le projet CSDP $^{2}$ [3-6], mis en œuvre en 1999, avait pour objectif de relancer la production de noix de coco, en appuyant le développement de plantations d'hybrides tolérant à la maladie en Central Region, et dans l'est de la Western Region. Ces hybrides à production rapide et importante de noix à haute teneur en huile, à condition d'appliquer l'ensemble l'itinéraire technique préconisé, devaient permettre aux producteurs d'être à nouveau compétitifs.

\footnotetext{
${ }^{1}$ L'usine d'Esiama, entre-temps renommée Vegetable Oil Mills et filiale du groupe Chana Industrial Holding Company (GIHOC), qui réunit des unités nationales de transformation de matière première, dut fermer ses portes en 1986. Cela faisait suite à la politique d'ajustement structurel conduite à partir de 1986, qui, en particulier, a proscrit les soutiens de l'État aux prix et a donc encouragé la concurrence de I'huile de coco avec celle d'autres oléagineux (palmier à huile).

${ }^{2}$ CSDP : Coconut Sector Development Project [3] financé par l'Agence française de développement, de 1999 à 2004, qui visait à diffuser I'hybride MYD $\times$ VTT au travers d'un paquet technologique (matériel végétal et engrais). Au total, près de 210000 plants distribués à plus d'un millier de producteurs et 1300 ha replantés.
}

Mais pour l'heure, les cocoteraies commencent seulement à produire, et les surfaces restent faibles.

Depuis cinq ans, les commerçants nigérians importent des noix matures en provenance de la Western Region. Cette activité s'est considérablement développée, en 2006, et a favorisé une forte augmentation du prix au producteur, qui, en 2008, est quatre à cinq fois supérieur à ce qu'il était début 2006.

\section{Préoccupations des principaux acteurs de la filière cocotier}

\section{Du côté des zones de production}

Les logiques de production des planteurs reposent principalement sur leurs possibilités d'accès aux marchés et bien évidemment sur le prix des noix. Ce facteur prix joue un rôle important dans l'affectation de la main-

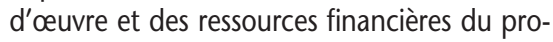
ducteur, qui sont liées aux revenus que cette activité procure [1]. Un espoir de prix meilleur est venu avec le marché au Nigéria, que beaucoup d'acteurs (chefs, propriétaires terriens et transformateurs, etc.) critiquent: certains disent que la filière traditionnelle procure plus de valeur ajoutée grâce à la valorisation des sous-produits; $d$ 'autres expliquent que le prix actuel amplifie l'intensité des vols de noix ou que ce marché est très volatil... La question est donc de savoir si ce marché peut être bénéfique à long terme.

\section{Du côté des transformateurs \\ de matière première}

(usine d'huile, de fibre, etc.)

L'ensemble des unités de transformation du secteur cocotier a des inquiétudes vis-à-vis de leur approvisionnement en matière première. Certaines sont déjà affectées par la pénurie causée par le développement de la maladie et l'affectation des noix à d'autres sous-filières (marché nigérian pour les noix débourrées, savonneries du Burkina Faso pour l'huile de coprah). Elles se demandent donc comment augmenter la production de noix ou comment elles peuvent nouer des partenariats durables avec les agriculteurs. Néanmoins, le marché de l'huile de coco reste très étroit et à faible potentiel. Le tissu d'entreprises de transformation locales est peu dynamique.

\section{Du côté du MOFA et de l'appui de la coopération française}

Le MOFA et ses partenaires se rendent compte que peu de résultats ont été apportés par les différents programmes d'appui à la filière. D'une part, la recherche sur la maladie, qui a commencé il y a plus de 70 ans, n'avance pas beaucoup, et les cocotiers hybrides distribués s'avèrent sensibles à la maladie, au moins dans certaines conditions. D'autre part, si le 


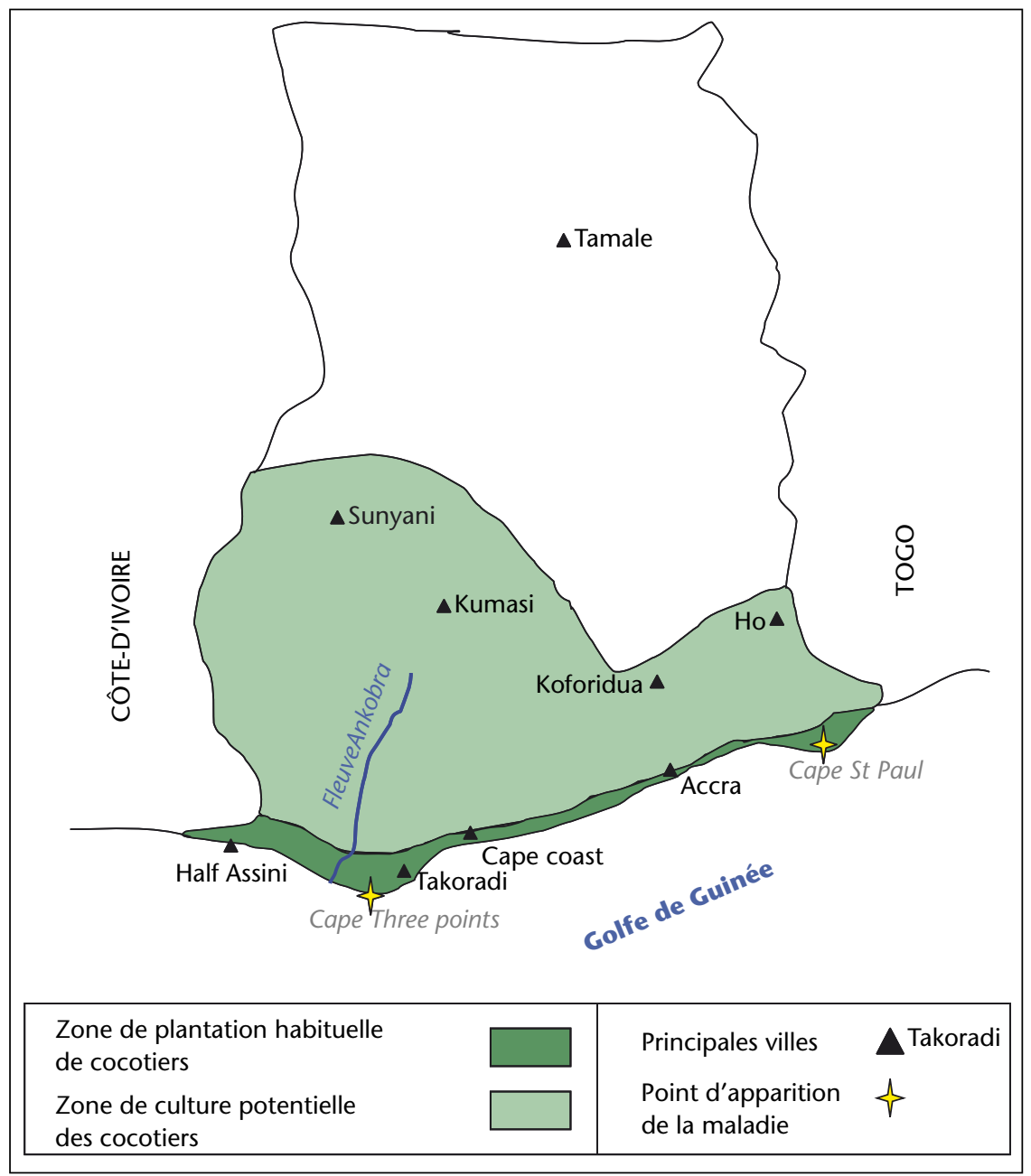

Figure 1. Zones de cultures potentielles et effectives du cocotier au Chana.

projet CSDP a peut-être permis d'augmenter un peu la production - ou au moins de la maintenir -, les agriculteurs n'ont pas trouvé, jusqu'en 2006 (date de l'arrêt du CSDP), de débouchés intéressant pour leur production. Les décideurs se demandent donc s'il faut continuer l'action ${ }^{3}$ dans ce secteur ou le laisser muter peu à peu vers d'autres cultures commerciales. Quant à l'intensification, elle ne pourra se faire que si la production est organisée de telle façon qu'elle rencontre un ou des marchés à haut potentiel en termes de consommateurs, de valeur ajoutée possible et de durabilité.

\footnotetext{
${ }^{3}$ L'arrêt de la coopération est lié à deux facteurs : l'absence de solutions solides à la maladie et le faible taux de remboursement des crédits octroyés aux producteurs partenaires du CSDP pour installer leurs plantations (inférieur à $20 \%$ des obligations contractuelles).
}

ment productives jusqu'à 70 ans, mais leurs rendements sont affectés par l'absence de restitution de la fertilité (absence de fertilisation). La majeure partie de la production d'huile de coco est vendue sur les marchés de gros, en général par des commerçantes originaires de la zone de production (principalement du district de Nzema East). Beaucoup de ces commerçantes sont également transformatrices. Celles qui n'assurent que la collecte préfinancent généralement la production. Les commerçants sont réunis dans une association appelée NZema Coconut Oil Producers and Traders Association, qui gère la vente sur les marchés de gros de Takoradi (Kokompe) et d'Accra (Agbogbloshie). Malgré la baisse de la production d'huile et donc la diminution du nombre de membres (500 en 2007 contre 1200 encore en 2003), cette association exerce le monopole sur le marché de gros de l'huile de coco.

Les détaillants viennent généralement s'approvisionner au marché de gros (figure 2). Les clients achètent l'huile de coco pour la cuisine domestique (sur la côte et surtout en Volta Region) ou la restauration. L'huile de coco est principalement utilisée pour la friture des aliments (poisson, igname, etc.). La consommation en corps gras augmente donc proportionnellement à la croissance démographique (la population ghanéenne a doublé en 25 ans) et à l'amélioration du pouvoir d'achat. Toutefois, cette évolution bénéficie peu à la filière huile de coco qui est concurrencée par d'autres types $\mathrm{d}^{\prime}$ huiles, en particulier les huiles raffinées industrielles (environ $40 \%$ de la consommation urbaine), qui se prêtent bien à la friture à haute température. L'huile de coco est davantage destinée aux consommateurs les moins riches. Donc, pour rester compétitif, le prix de I'huile de coco doit rester inférieur à celui de I'huile raffinée (en 2007, il était d'environ 0,6 contre $0,8 \mathrm{Chc}$ /bouteille pour l'huile raffinée). Malgré un prix apparemment croissant, dû à la conjoncture actuelle du secteur oléagineux, sa valeur réelle a peu augmenté par rapport aux autres huiles (figure 3). La demande d'huile de coco occupe une place de plus en plus faible dans la demande globale d'oléagineux. Cette demande reste néanmoins assez stable, car I'huile de coco occupe une place non négligeable dans les habitudes alimentaires des habitants de la zone littorale et de la Volta Region. II est peu envisageable que l'huile de coco concurrence les autres secteurs oléagineux dans le court et moyen termes. La stagnation inéluctable de la production de noix de coco (le rythme de replantation est très faible) contraindra obligatoirement les consommateurs vers d'autres produits. De plus, les performances technicoéconomiques par hectare de la cocoteraie sont très inférieures à celles $d u$ palmier à huile. 


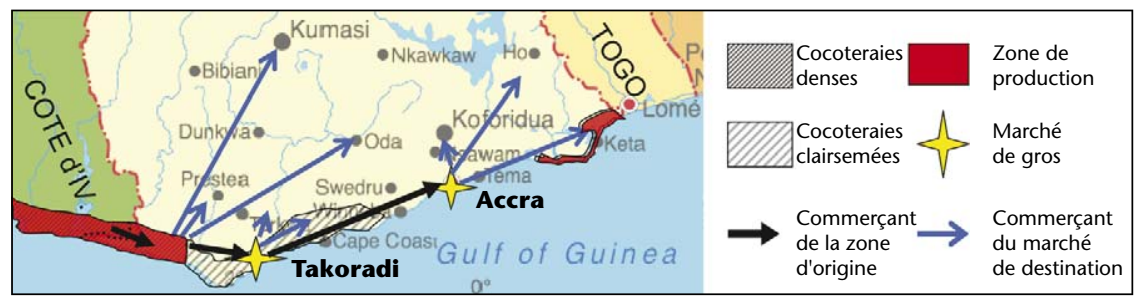

Figure 2. Modélisation spatiale de la filière huile de coco.

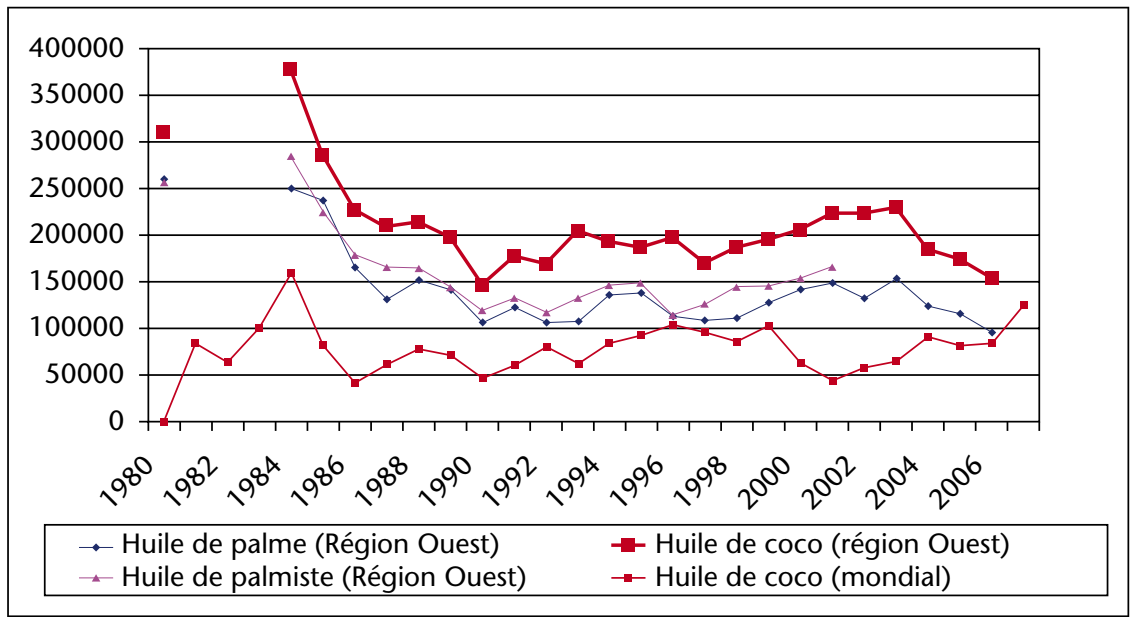

Figure 3. Évolution des prix de I'huile en région Ouest pour 4 gallons, en cedis 2006.

\section{Sous-filière noix débourrées : un gros potentiel}

Les seules chances de I'huile de coco résident donc dans la valorisation de ses qualités intrinsèques : goût unique et bénéfices pour la santé, qui pourraient lui permettre d'être vendue à un prix élevé, au Ghana ou à l'étranger. Mais cela implique un effort important d'organisation pour la relance de l'ensemble de la filière.
Les noix utilisées dans cette sous-filière proviennent principalement de l'extrême-ouest de la côte ghanéenne, c'est-à-dire de la même zone que pour I'huile de coco. En effet, les noix pelées doivent pouvoir être transportées, et donc être résistantes aux chocs et se conserver longtemps. Cela disqualifie les variétés naines ou hybrides, à germination rapide, et privilégie la variété locale WAT, qui ne se retrouve en quantité importante que dans cette zone (figure 4).

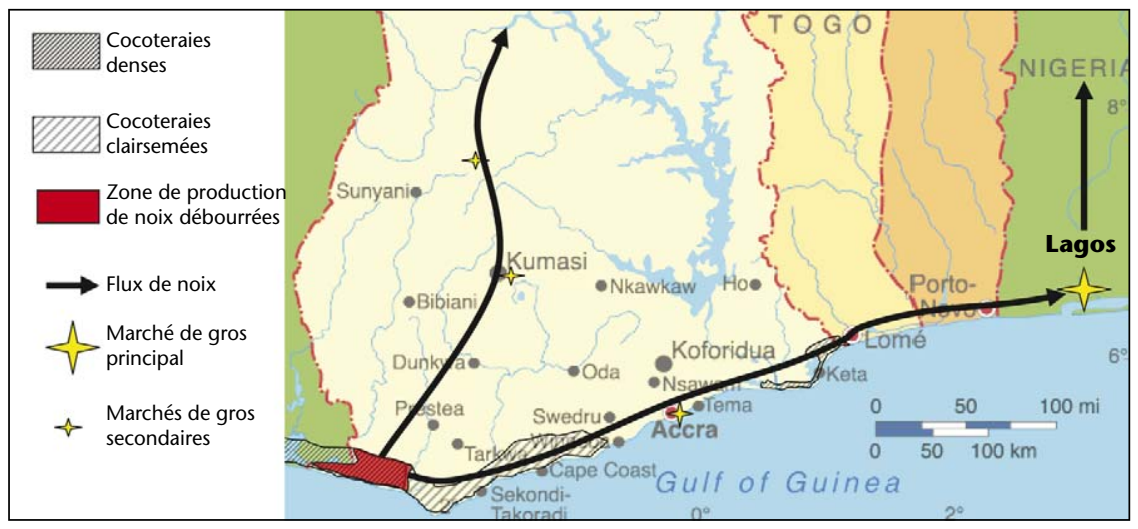

Figure 4. Modélisation spatiale de la filière noix débourrées.
La plupart des noix matures sont vendues aux nigérians, mais une partie peut être vendue à des usines ghanéennes, ou à des commerçants ghanéens, possédant suffisamment de capital financier pour les exporter sur les marchés du nord du Ghana.

Depuis 2002, des commerçants nigérians ont commencé à importer des noix pelées du district de Jomoro. Les quantités collectées, faibles au départ, ont fortement augmenté à partir de 2006 pour atteindre aujourd'hui près de la moitié de la production de la zone ouest. Les grossistes, basés à la frontière du Nigéria (zone de Badagry), emploient des agents qui s'installent dans sur la zone de production (Tikobo) pour collecter les noix. Ces agents sont de jeunes nigérians qui travaillent le plus souvent en binôme (ou trinôme), ont pour objectif de charger un camion à échéance régulière (hebdomadaire, quinzaine ou mensuelle)... Au Nigéria, les grossistes vendent les noix par sacs de 40 unités à des intermédiaires de Badagry, qui les exportent vers d'autres régions du Nigéria (certaines noix migrent jusqu'au Niger), en particulier le nord (Abuja) et l'est du pays. Une autre partie des noix est revendue à des commerçantes de rue de Lagos ou à des entreprises locales (cosmétique ou biscuiterie). Les noix de coco sont utilisées pour préparer le " cocorice ", être consommées telles que ou avec du gari ${ }^{4}$. Les noix de variété WAT ghanéennes sont appréciées et reconnues au Nigéria pour leur saveur sucrée.

Au Ghana, une proportion réduite des noix matures pelées est vendue sur les marchés des grandes villes, principalement celles du nord du Ghana. Les détaillants vendent les noix par lot de quatre ou cinq ou tout simplement des morceaux d'albumen aux passants. Des noix matures sont aussi vendues à un prix élevé ${ }^{5}$ à des usines préparant des cacahuètes enrobées. La demande de noix matures est très forte tant au Nigéria que dans l'ensemble des pays d'Afrique de l'Ouest (achat de noix au Bénin, Togo, Côte-d'Ivoire, Burkina Faso). Cette pression sur la demande est durable (production stagnante) et se répercute sur le prix de la noix de coco. Le prix actuel au producteur ghanéen $(0,2 \mathrm{Ghc} /$ noix $)$ est multiplié par trois pour les consommateurs de Lagos. La croissance démographique, en particulier au Nigéria, abonde en faveur d'une demande de plus en plus forte. Le prix de la noix de coco restera élevé, excepté si le gouvernement ghanéen impose un gel des exportations pour protéger

\footnotetext{
${ }^{4}$ Le gari est une poudre grillée de manioc fermenté, souvent consommée par les populations pauvres.

${ }^{5}$ Ces usines sont en mesure de proposer un prix élevé, car les noix ne représentent qu'une faible partie du coût de production.
} 
la filière locale d'huile de coco, mais aussi préserver les intérêts des chefferies de la Western Region. Une autre condition au maintien de la noix mature ghanéenne sur le marché nigérian est le développement des plantations de WAT locale, ou une autre variété aux caractéristiques similaires (germination lente, coque résistante), mais moins sensible à la maladie.

L'usine WIENCO, basée à Tikobo, valorise les sous-produits de la noix mature: la bourre (fabrication de cordes, de tapis, etc.) et la coque (cendres entrant dans la fabrication des filtres, etc.). Cette usine emploie 100 personnes. WIENCO éprouve d'énormes difficultés pour acheter des coques qui sont aujourd'hui principalement exportées au Nigéria. WIENCO avait pour projet d'appuyer le développement de plantations villageoises sous forme contractuelle, pour sécuriser ses approvisionnements. La pression sur les prix rend ce scénario peu réaliste, car WIENCO ne peut s'aligner au niveau des commerçants nigérians. Une alternative possible pour WIENCO serait de développer la production d'huile de coco pressée à froid qui est rémunératrice. L'investissement nécessaire pour développer une unité de pression à froid est modéré. Cela pourrait offrir à WIENCO la possibilité de proposer un prix producteur compétitif.

\section{Sous-filière coprah : une filière complémentaire, à potentiel limité}

II s'agit de la production d'huile par la voie sèche, qui est la plus courante à travers le monde. Les transformateurs ghanéens privilégient la transformation d'huile par voie humide, bien que les industries favorisent la voie sèche, plus performante. Au Ghana, I'huile de coprah est produite à partir des noix matures pelées, rejetées par les commerçants nigérians (calibre trop petit) ou de coprah importé de Côte-d'Ivoire. L'extraction d'huile de coprah au Ghana est principalement industrielle (l'extraction artisanale est surtout destinée à la fabrication artisanale de savons). Aujourd'hui, les usines ont presque toutes des difficultés à s'approvisionner en coprah. Certaines entreprises ont des partenariats avec des commerçants ghanéens qui les livrent régulièrement, mais la plupart s'approvisionnent en Côte-d'Ivoire. Mais le coprah ivoirien est devenu très cher, à cause d'une parité franc CFA-cédis fortement défavorable aux importations, mais aussi des fortes taxes à l'entrée. Ces usines tentent donc de se reporter sur $d^{\prime}$ autres alternatives (palmistes, arachide, soja, etc.). La plupart des usines vendent directement leur production à des clients réguliers (figure 5).

La plupart des savonneries industrielles ghanéennes n'utilisent plus d'huile de coprah.

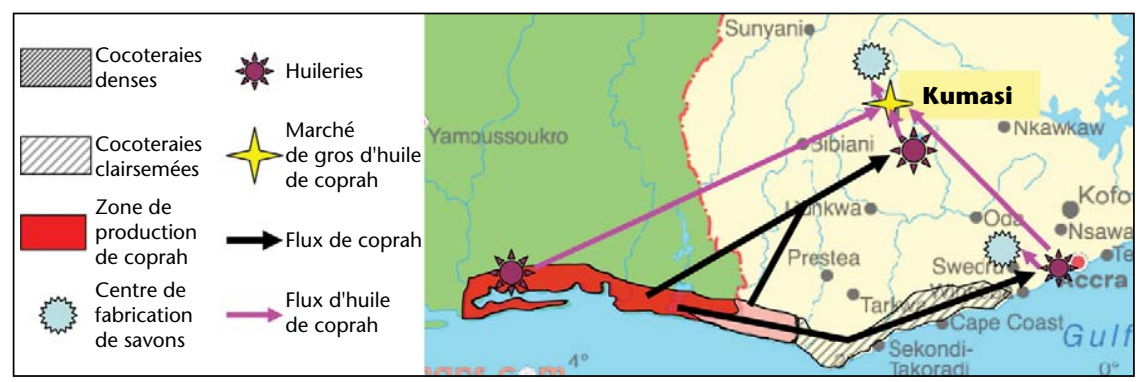

Figure 5. Modélisation spatiale de la filière d'huile de coprah.

D'une part, l'approvisionnement en huile de coprah n'est pas suffisant, d'autre part, I'utilisation de l'huile de palme et de la soude permet $\mathrm{d}^{\prime}$ obtenir du savon à bas prix. La saponification traditionnelle avec I'hydroxyde de potassium se prête bien à la fabrication de savon liquide. Mais sa production reste faible, fortement concurrencée par les savons liquides importés, et pénalisée par le coût élevé des parfums importés (seul le parfum citron est synthétisé au Ghana). Le potentiel du marché du savon reste donc limité. Toutefois, cela ne devrait pas être suffisant pour que se développe une production spécifique de coprah.

\section{Filière noix fraîches :}

une petite filière bien valorisée

avec une faible concurrence

Les noix fraîches sont des noix récoltées encore immatures (neuf mois après fécondation) principalement pour la consommation de l'eau de coco. Les noix fraîches sont vendues dans les grandes villes, où elles arrivent entières. Elles sont donc lourdes et volumineuses, ne se conservent pas longtemps, et leur coût de transport est élevé. La plupart des noix fraîches proviennent des zones côtières de la région Centrale et de l'est de la région Ouest (zone de Takoradi). On note un début de développement de plantations à proximité des pôles de consommation, qui sont principalement Accra et Kumasi. Si, aujourd'hui, la plupart des noix fraîches mises en marché sont de la variété locale WAT, les noix d'hybrides qui ont un gros volume d'eau sont très appréciées. Cependant, elles se conservent plus difficilement (elles peuvent s'abîmer en moins d'une semaine, contre un mois pour la variété locale). Les noix fraîches représentent un débouché très intéressant et rémunérateur pour les producteurs situés non loin des pôles urbains, mais également pour ceux qui ont planté des hybrides. Le nombre de consommateurs de noix fraîches est difficile à estimer, mais reste encore assez faible. Le développement de cette sousfilière est lié à l'amélioration du niveau de vie urbain (figure 6).

\section{Bilan : quelle(s) filière(s) possible(s) pour relancer le secteur de la noix de coco ?}

La sous-filière qui répondra le mieux aux préoccupations des producteurs est celle qui valorisera le mieux leur production par un prix d'achat élevé.

La sous-filière huile de coco n'est potentiellement intéressante que pour le développement local et persiste principalement grâce aux pratiques d'usure (les noix sont gagées avant d'être récoltées). Son marché reste de plus en plus aléatoire, car pour s'approvisionner les transformateurs locaux devraient s'aligner sur les prix de Nigérians, sans pour autant augmenter le prix de vente de l'huile, ce qui est impensable. Le développement de cette sous-

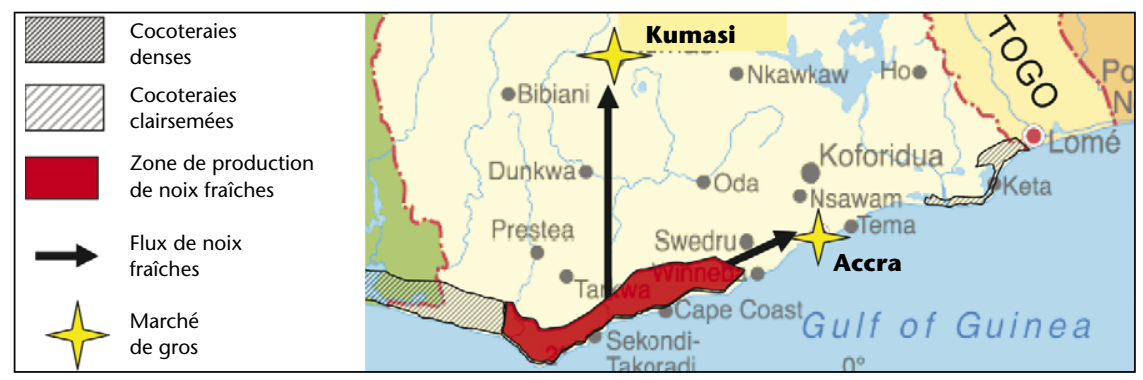

Figure 6. Modélisation spatiale de la filière noix fraîches. 
filière repose sur les créneaux de qualité (pression à froid par voie humide), mais il ne pourra impliquer que des opérateurs hautement professionnels.

La sous-filière huile de coprah est aujourd'hui marginale au Ghana. Les importations de coprah de Côte-d'Ivoire ont néanmoins permis de créer de la valeur ajoutée et de l'emploi urbain. Le développement de cette sousfilière, au même titre que celui de l'huile de coco vierge pressée à froid, pourrait avoir un intérêt dans le cadre d'un scénario d'intégration de différents segments de la filière (production de noix matures, valorisation de la bourre et de la coque, transformation, etc.). Cela permettrait de produire plus de valeur ajoutée (collecte, cracking, transport, etc.), et de développer l'emploi urbain (transformation, mise en marché, etc.).

La sous-filière noix fraîches est celle qui produit le plus de valeur ajoutée (surtout en ville). Son marché, aujourd'hui limité, pourrait être stimulé en favorisant le développement des cocoteraies périurbaines (ce qui est déjà le cas à la périphérie de Kumasi). La proximité devrait résoudre les contraintes de conservation et le poids du coût de transport.

La sous-filière noix matures débourrées pour l'exportation au Nigéria est celle qui a le meilleur potentiel économique, tant pour le producteur que pour les grands agrégats nationaux. Cette sous-filière sera d'autant mieux valorisée qu'une part plus importante de la valeur finale sera produite au Ghana, en particulier en valorisant les écarts de triage (petites noix pour la production d'huile, etc.), la bourre et la coque.

\section{Quels appuis apportés à la relance de la filière noix de coco?}

La relance de la filière est liée à deux facteurs, l'existence d'un marché et la volonté des producteurs à produire plus. Aujourd'hui, le constat est que la noix de coco est un produit aux usages multiples qui bénéficie de nombreuses opportunités de marché. L'évolution rapide du prix (qui a quasiment triplé pour les noix sèches en 2006) a modifié la position de la filière maintenant plus attractive, mais aussi de la place de la cocoteraie au sein des systèmes de production. Ces transformations économiques ont également un impact important sur les rapports sociaux. Un certain nombre d'ateliers de transformation, dont certains étaient liés aux chefferies, assuraient leurs approvisionnements grâce à des pratiques d'usure élaborées. Les prix réels d'achat qui étaient très bas, entre autres ont permis de maintenir les prix de l'huile de coco à des niveaux inférieurs à ceux des huiles concurrentes (en achetant les noix à des prix très bas, taux d'intérêt oblige). Nous essayerons de voir quelles sont les opportunités pour les producteurs et pour la filière et de définir comment on pourrait en tirer parti.

\section{Des producteurs motivés à produire davantage}

L'agriculteur est bien évidemment au centre des stratégies de relance de la production. Cet axiome simple ne doit pas masquer la complexité du sujet. L'histoire du secteur agricole a démontré que les exploitations ont toujours fait preuve d'une réelle capacité d'adaptation pour soutenir leurs activités et tenter de préserver leurs conditions de vie face à un environnement économique instable, l'étiage de l'offre des marchés et des services, mais aussi les facteurs de risques récurrents qui affectent les exploitations. Cette capacité d'adaptation est un atout, mais elle détermine également la nature de cheminement élaboré qu'opère un producteur pour s'engager dans une logique de modernisation. Les tenants de son raisonnement reposent principalement sur l'appréciation des résultats supplémentaires qu'il tirera et des risques qu'il évacuera en changeant ses pratiques. Cette position est très variable, en fonction à la fois de sa situation (capitaux disponibles, etc.), des réalités de son environnement physique et institutionnel (disponibilité des services, etc.), des opportunités qui prévalent (prix, marchés, accès aux subventions, etc.) et de son cadre social (organisation sociale, règles foncières, gestion des ressources, appartenance à des $\mathrm{OP}$, etc.). La matrice de prise de décision économique, à la suite (figure 7), illustre la complexité des mécanismes d'appui à mobiliser, quand on centre des stratégies sur les exploitations agricoles familiales (cf. matrice 1). Cette complexité n'est pas à négliger dans la mise en place de stratégies de progrès, même si le facteur prix reste déterminant.

\section{Pourquoi les producteurs sont plus motivés aujourd'hui à produire?}

Les études relatives aux évolutions du revenu d'une cocoteraie par hectare, réalisées par le bureau Institutions et Développement en 2006, révèlent l'ampleur des changements qui ont affecté les exploitations au cours de cette année. Cette étude a essayé de comparer les modifications du revenu des producteurs,

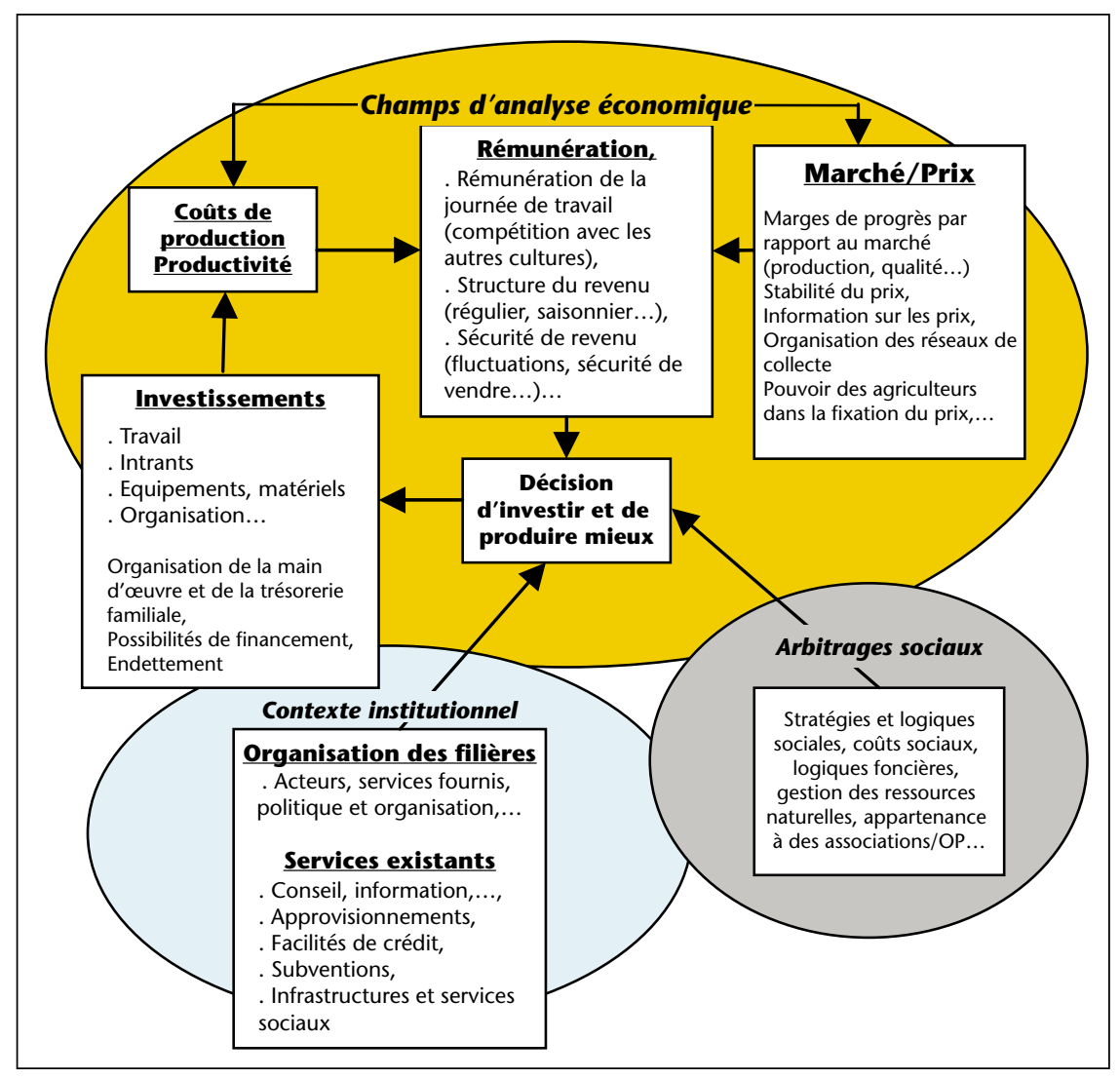

Figure 7. Matrice de prise de décision économique. 
en prenant comme témoin une plantation traditionnelle, autour de quatre scénarios : fertilisation sur plantation traditionnelle, plantations d'hybrides en considérant des seuils de production correspondant à 100, 90 et $80 \%$ du rendement optimal de la variété (figures 8 et 9 ). II présente donc les résultats sur la base de trois hypothèses de prix concernant la vente de noix sèches $(30000,50000$ et 60000 cedis/100 noix) et deux hypothèses pour la vente de noix fraîches (50000 et 70000 cedis/100 noix). Les valeurs de prix correspondent aux moyennes de prix pratiquées par les acheteurs durant le premier semestre 2006 (20 à 30000 cedis/100 noix sèches, 40 à 50000 cedis/100 noix fraîches), et le second semestre $2006 \quad(50,60$, voire 70000 cedis/100 noix sèches, 70000 cedis/ 100 noix fraîches). La demande du marché nigérian a brutalement explosé à partir du mois de mai 2006.

Ces estimations montrent que l'intérêt de l'intensification et de la replantation est évident durant les cycles où les prix sont élevés. La marge la plus importante est dégagée par la vente de noix fraîches. Les besoins en main$\mathrm{d}^{\prime} œ u v r e$ sont moins importants dans ces dernières plantations qu'ils ne le sont pour les plantations qui récoltent en sec. Aujourd'hui, l'intérêt économique du producteur est donc d'améliorer ses techniques de production. Le revenu d'une exploitation de noix de coco planté en hybrides et dont la production est vendue fraîche pouvait atteindre fin 2006, 14478000 cedis/ha par $a^{6}$.

Cette étude s'est également interrogée sur le poids des investissements nécessaires pour atteindre les meilleurs résultats économiques possibles. II s'agissait de comprendre quelle est la part des coûts de production par rapport au chiffre d'affaires (figure 10).

Ces coûts de production se distribuent entre l'achat d'intrants (engrais, pesticides), et l'achat de main-d'œuvre (maintenance de la plantation, transport, etc.). Les charges de main-d'œuvre et les achats d'intrants ont un poids comparable dans les exploitations qui vendent la noix fraîche (figure 11). La part de la main-d'œuvre occupe entre 70 et $100 \%$ des coûts de production des exploitations qui commercialisent en sec (figure 12).

Le coût de la main-d'œuvre dans les villages est cher (30000 cedis/j), et sa productivité est faible. Cette main-d'œuvre devient également de plus en plus difficile à trouver. De plus en plus de chefs d'exploitation recourent à des prestations extérieures qui nécessitent de mobi${ }^{6}$ Équivalent de 1448 Chc. 1 Ghc $=10000$ cedis
(ancien cedis).

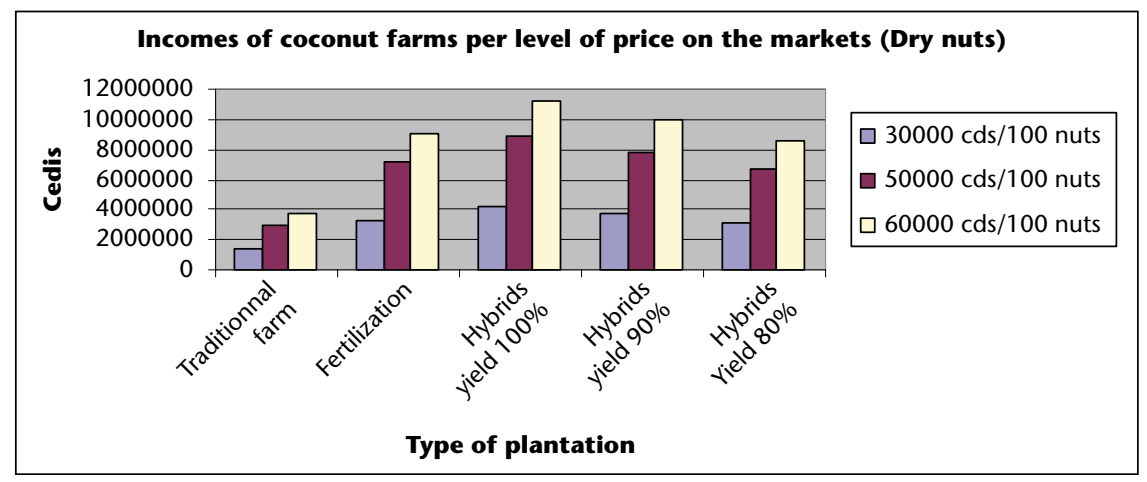

Figure 8. Revenu d'exploitation d'un hectare de plantation par niveau de prix (noix sèches).

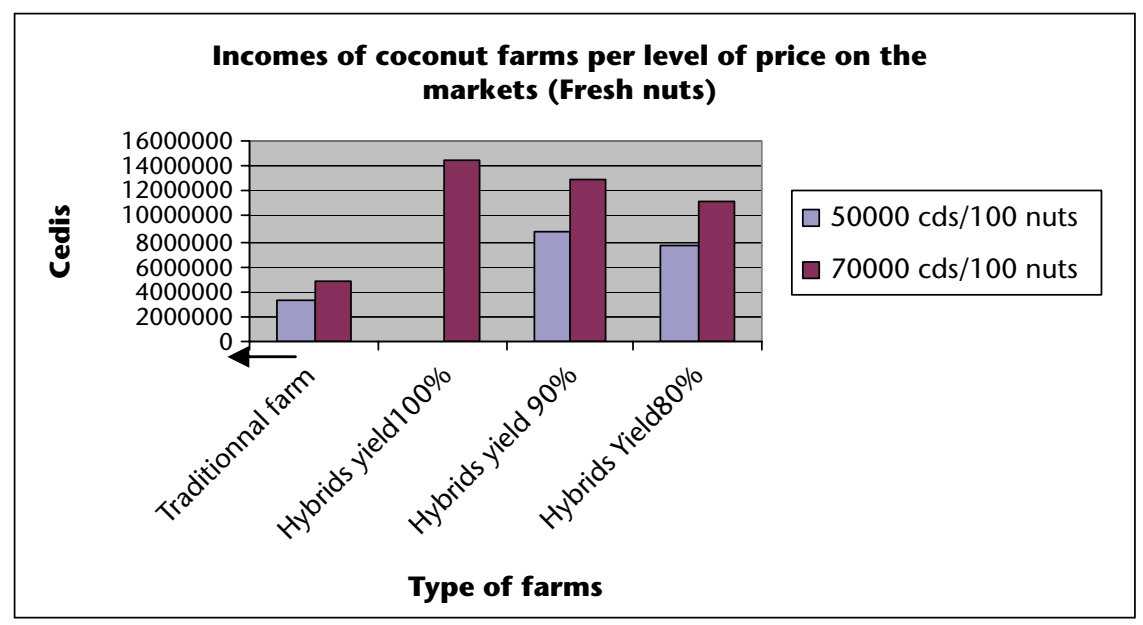

Figure 9. Revenu d'exploitation d'un hectare de plantation par niveau de prix (noix fraîches).

\section{Operational costs per type of coconut farm and per level of price (Fresh and dry nuts)}

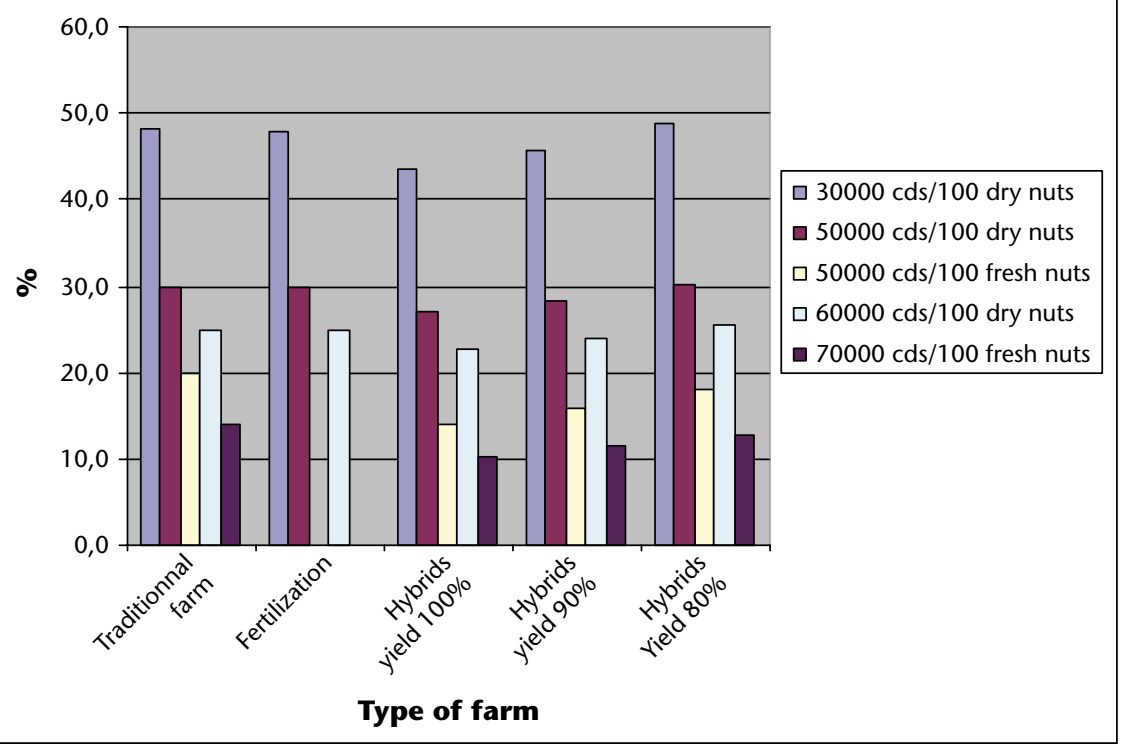

Figure 10. Part des coûts de production par type de système de production et par niveau de prix. 


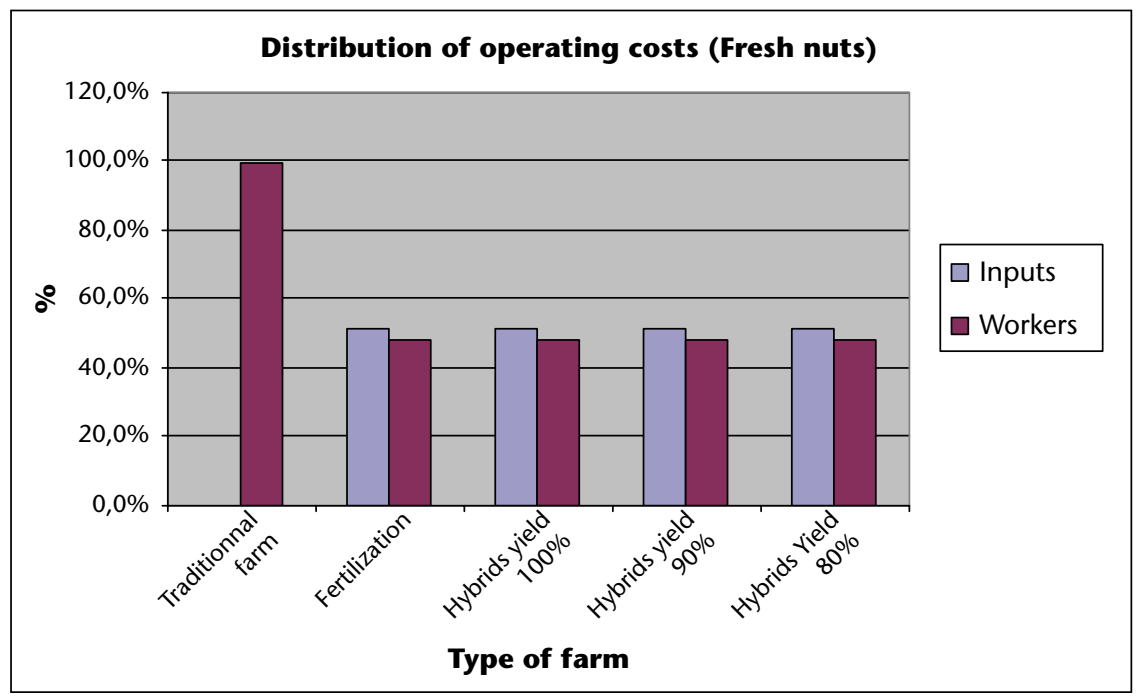

Figure 11. Distribution des coûts de production sur une exploitation qui commercialise la noix fraîche.

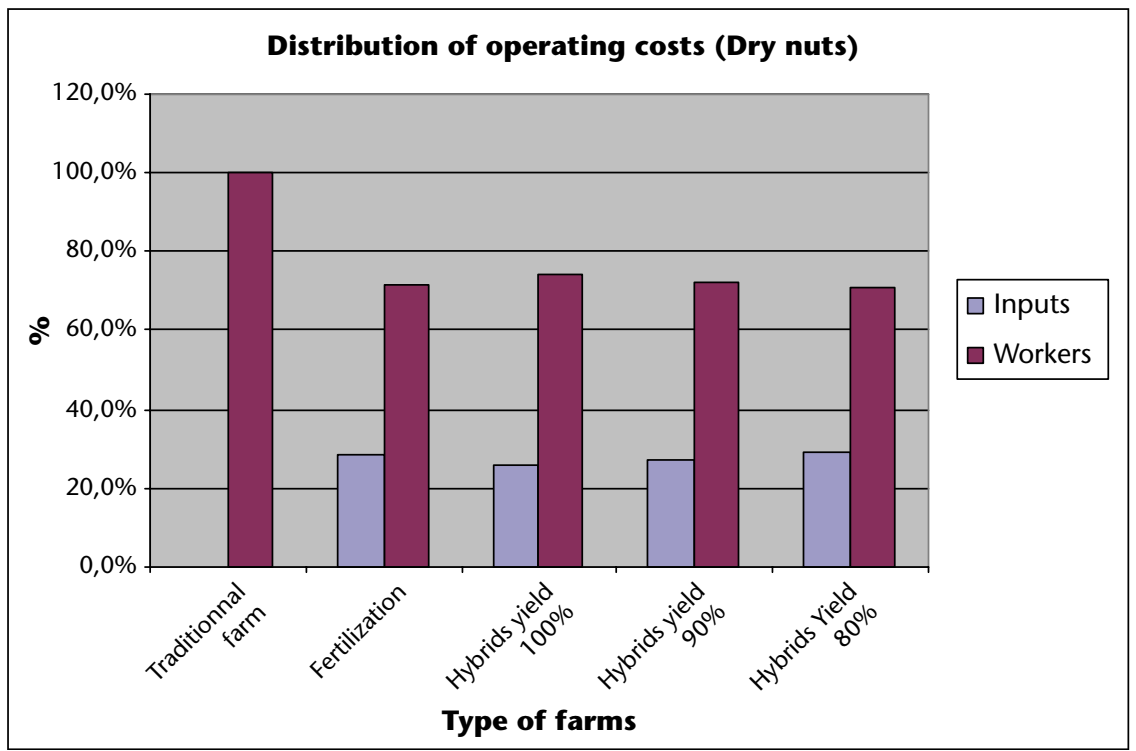

Figure 12. Distribution des coûts de production sur une exploitation qui commercialise la noix sèche.

liser une trésorerie conséquente. Cela met le producteur en situation de fragilité. S'il ne peut mobiliser cette main, celle-ci est prise en charge par les acheteurs à un prix plus élevé. De même, cette difficulté à mobiliser de la main-d'œuvre influe sur la qualité des entretiens des plantations. L'enquête menée par Institutions et Développement auprès des producteurs fait apparầtre que $30,5 \%$ des plantations sont très bien tenues et que $20,2 \%$ d'entre elles sont très mal tenues. Le développement des services financiers de proximité dans la zone pourrait remédier à ces absences de trésorerie, mais les producteurs de noix de coco n'y ont pas bonne presse aujourd'hui.

hevéa est intégrée par une société hévéicole). Le CSDP a fourni cing grands types de services aux agriculteurs :

- vulgarisation en s'appuyant sur des groupes de base qui, aujourd'hui, sont constitués en une organisation de producteurs faîtière de la filière : la COFPMAG' ${ }^{7}$. Les techniques de production sont aujourd'hui bien maîtrisées par les agriculteurs, mais n'ont été que partiellement appliquées (en particulier les entretiens). Les prix de la noix de coco sont effectivement restés bas jusqu'en mai 2006 et ne justifiaient pas d'investissements importants pour les planteurs ;

- approvisionnements en intrants et appui technique à la mise en place des plantations ;

- appui à la négociation de lignes de crédit avec l'agence bancaire ADB de Takoradi, au bénéfice des producteurs volontaires, pour mettre en place les plantations (paiement des intrants et des travaux de préparation des plantations);

- appui à la structuration des producteurs en vue de mettre en place une organisation qui assure la relève des activités du projet à son retrait (mise en place de la COFPMAG) ;

- appui à la mise en place d'une pépinière fonctionnelle (gérée par la COFPMAG).

Le projet n'avait pas intégré de volet d'appui à la commercialisation (les producteurs étaient très mal informés sur les opportunités du marché et sur les prix). Un appui a également été apporté au secteur de la recherche. L'arrêt du projet a été conditionné par deux facteurs :

- les hybrides développés par la recherche ne donnent pas encore la garantie d'être résistants à la maladie ;

- le faible taux de remboursement des producteurs (figure 13).

La faiblesse des taux de remboursement a fortement pesé dans la décision d'interrompre ce programme. Plusieurs raisons ont influé sur le faible niveau de remboursement des crédits par les planteurs :

- le mauvais état de nombreuses plantations, lié au faible intérêt économique de l'activité jusqu'en 2006 ;

- ce mauvais état des plantations traduit également la sociologie des producteurs de noix de coco (qui reste avant tout la culture des ménages en difficulté). L'enquête conduite par Institutions et Développement auprès de 570 « nouveaux » planteurs, en 2006, montre que $60 \%$ d'entre eux ont plus de 50 ans et $84,5 \%$ d'entre eux n'ont pas suivi le second cycle scolaire. La plupart de ces planteurs vivent dans un état $d^{\prime}$ endettement chronique ; - les « délais de grâce » de deux ou trois ans que I'on a proposé aux agriculteurs pour entamer leur remboursement. Beaucoup d'exploitants avaient oublié leurs engagements quand on leur a demandé de rembourser, d'autres considéraient l'appui du projet comme une subvention, etc :
${ }^{7}$ Coconut Farmers, Processors and Marketers Association in Chana. 


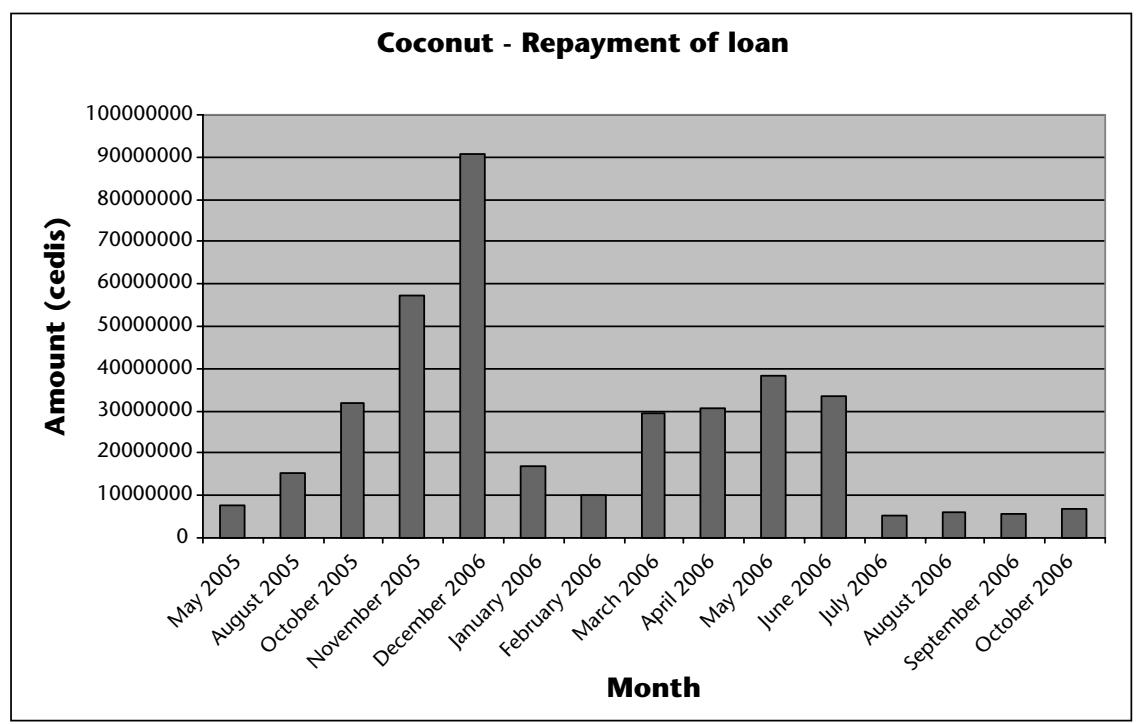

Figure 13. Niveau de remboursement des crédits par les agriculteurs.

- l'absence de suivi des remboursements par la banque commerciale ADB. Cette démission a été fortement préjudiciable au programme. D'une part, les paysans ne se sont pas sentis engagés, car cette banque n'a produit aucun document précisant la situation du crédit de leurs clients. D'autre part, le suivi et la collecte des remboursements ont été confiés aux agents vulgarisateurs et socio-organisateurs du CSDP, que les paysans fuient maintenant (donc les messages ne passent plus).

La figure 13 montre également que les remboursements ont fortement chuté avec l'annonce d'un arrêt probable du projet. La question cruciale du remboursement a également modifié la position des planteurs visà-vis de la COFPMAG, qui aujourd'hui leur pose plus de problèmes qu'elle n'en résout. Elle est en charge du recouvrement. La plupart d'entre eux ont déserté l'association qui ne dispose plus des ressources issues des cotisations pour mener ses activités.

\section{Une relance à quelles conditions?}

Le développement de la filière noix de coco ne peut être dissocié de la question du marché. Le facteur prix intervient très sensiblement dans la décision des agriculteurs de mettre en œuvre les pratiques conseillées. Les conditions favorables de prix et de marché existent, mais le secteur de la production est aujourd'hui déstructuré, et isolé d'un certain nombre de services dont il a encore besoin (conseil technique, approvisionnement en matériel végétal, surveillance épidémiologique, etc.). Dans le district de Jomoro, l'entreprise WIENCO souhaite soutenir les planteurs qui veulent produire plus et mieux, mais, à l'opposé du secteur hévéa, il est impossible qu'une entreprise agroindustrielle structure à elle seule l'ensemble d'une filière aussi complexe. La COFPMAG gère une pépinière fonctionnelle avec l'appui de techniciens de l'agriculture, mais cela reste très insuffisant par rapport à l'ampleur actuelle de la demande en hybrides.

En fait, le déclenchement d'un processus d'appui au développement de la filière repose sur quatre questions clés :

- les solutions de la recherche donnent-elles aujourd'hui des garanties à l'agriculteur pour risquer un investissement « lourd ", face à une maladie du jaunissement mortel qui reste encore très imprévisible? Quelle est la durée de vie probable d'un hybride sans risquer une infection? Les mesures de containment testées donnent-elles des garanties suffisantes pour contrôler les risques de contamination? Un atout certain pour la filière est que le prix actuel de la noix de coco permet un retour sur investissement rapide ;

- à l'heure actuelle, le marché nigérian « porte » un prix rémunérateur, qui le restera probablement durablement, vu le déséquilibre actuel entre l'offre et la demande. Cette situation peut évoluer au détriment du planteur si le gouvernement ghanéen gèle les exportations de noix de coco vers le Nigéria. Une position politique durable doit être arrêtée ;

- I'arrêt du CSDP induit un risque de stagnation de la production d'hybrides. II n'est pas envisageable de raisonner la relance sans la garantie de pouvoir produire le matériel végétal de base approprié ;

- la capacité réelle de la COFPMAG à acquérir une réelle légitimité auprès des planteurs, à four- nir des services de proximité (vulgarisation, conseil, information, etc.), à assurer la surveillance sanitaire, à proposer des mesures adaptées, et à représenter les producteurs dans les débats de la filière et vis-à-vis du gouvernement ?

\section{Pas de relance de la filière sans vision}

La filière a longtemps été considérée comme marginale par rapport à d'autres secteurs de l'économie agricole. La donne a changé : la noix de coco développe de la valeur ajoutée et de l'emploi tant à l'échelle locale, que nationale (WIENCO, industries de traitement du coprah, transport, manutention, etc.) et est aujourd'hui un vecteur important $d$ 'intégration économique régionale. Il est important de faire reposer les programmes de relance de la filière sur une stratégie à moyen terme (politique filière) concertée qui engage les trois parties (État, producteurs, secteur privé, etc.) autour d'une vision claire et partagée, d'un plan d'affaire qui précise engagements respectifs et objectifs de résultat. Cette vision reposerait sur trois engagements principaux :

- celui de l'État à promouvoir le secteur, en particulier en :

- soutenant la recherche agronomique,

- garantissant la production d'un matériel végétal adapté, résistant et en quantité suffisante,

- et en organisant en partenariat avec la profession et le secteur privé, la surveillance épidémiologique de la cocoteraie, et en veillant à I'application des mesures de contrôle de la maladie retenues (containmant). Des acteurs de la filière bien organisés doivent être en mesure de contrôler le développement de la maladie ;

- celui de la profession agricole. Le rôle que I'on pourrait accorder aux organisations de producteurs est triple :

- mettre en place les services de proximité dont ont besoin leurs membres pour améliorer leur productivité (conseil, accès aux intrants, formation, etc.) et leur faciliter l'accès aux marchés (information, mise en relation, appui à la contractualisation, etc.),

- assurer la représentation de la profession au sein de la filière, en particulier, mieux organiser les relations avec le secteur agro-industriel, - gérer les procédures de contrôle de l'extension de la maladie en étroite relation avec les services déconcentrés du MOFA ;

- celui du secteur privé repose sur la recherche d'une meilleure complémentarité entre les différents sous-secteurs de la noix de coco, pour soutenir le secteur de la production (WIENCO...) et favoriser la création d'un maximum de valeur ajoutée au Ghana.

En dernier lieu, le dossier brûlant du remboursement des crédits octroyés par l'ADB doit être rapidement apuré, cela de manière définitive, 
en considérant bien les responsabilités respectives des différentes parties prenantes. Le secteur noix de coco nécessite des investissements qui ne pourront se faire que dans un climat totalement clarifié et assaini.

\section{Conclusion}

Le développement de la filière noix de coco et les revenus des producteurs sont étroitement liés aux mesures qui seront prises pour maintenir des niveaux de prix attractifs, contenir l'extension de la maladie du jaunissement létal et pouvoir mettre en place les conditions d'investissement nécessaires au développement du secteur de la production (matériel végétal, crédit, etc.). Cela implique de concrétiser trois préoccupations centrales :
- avoir des producteurs organisés au sein de la filière qui arrivent à prendre en main un certain nombre de services de proximité nécessaires à leurs exploitations ;

- avoir une filière suffisamment structurée qui permet le dialogue entre ces acteurs et l'État ;

- mais surtout, décliner une politique qui permet à l'ensemble de ces acteurs de s'engager par rapport à une vision commune claire.

\section{RÉFÉRENCES}

\section{Bene 2006.}

2. Ruf F. «French project » contre « French project » au Ghana. CIRAD, UMVR, novembre 2006, 15 pages.
3. CSDP. Coconut marketing in Chana-Training workshop report. Tikobo, octobre 2006, 21 pages.

4. CSDP. Institutions et Développement. Support to the ROAA and the COFPMAG. Mission report September-October 2006, ROPP, octobre 2006, 96 pages.

5. CSDP. Institutions et Développement. Support to the ROAA and the COFPMAG. Mission report August 2006, ROPP, septembre 2006, 61 pages.

6. CSDP. Institutions et Développement. Support to the ROAA and the COFPMAG. Mission report November-December 2006, ROPP, décembre 2006, 55 pages.

7. Salinier J. Comment orienter le secteur cocotier ghanéen pour qu'il contribue mieux au développement. Mémoire ITARC, décembre 2007, 196 pages. 